\title{
Creatine kinase activity in patients with brittle asthma treated with long term subcutaneous terbutaline
}

\author{
A P Sykes, N Lawson, J A Finnegan, Jon G Ayres
}

\begin{abstract}
Infused beta $a_{2}$ agonists have been shown to cause focal myocardial necrosis. Serum creatine kinase activity was compared in 13 patients with brittle asthma currently being treated with subcutaneous terbutaline and an age and sex matched control group of patients with moderate asthma having inhaled treatment only. The median serum total creatine kinase activity for patients receiving subcutaneous terbutaline (211 units/1) was greater than that for the control group (120 units/1). The cardiac specific isoenzyme component of creatine kinase was not raised in either group, and the electrocardiograms and serum aspartate aminotransferase activity were normal. Electromyograms in five patients receiving subcutaneous terbutaline with high creatine kinase activity showed changes consistent with myositis in two, one of whom was subsequently shown to have a metabolic myopathy, which is thought to be long standing. No pathological changes were seen in the myocardium at necropsy in a patient who died from an acute attack of asthma while taking subcutaneous terbutaline. These results suggest that the raised creatine kinase activity seen in patients receiving this treatment is unlikely to be myocardial in origin.
\end{abstract}

High circulating concentrations of the catecholamines noradrenaline and adrenaline may cause acute focal myocarditis, both experimentally in animals ${ }^{1-5}$ and clinically in man, especially in patients with phaeochromocytoma. ${ }^{156}$

Kurland et $a l^{7}$ reported focal myocardial necrosis in a young patient with asthma who died during an acute attack of severe asthma treated with subcutaneous adrenaline and intravenous isoprenaline and aminophylline. Acute focal myocarditis has also been reported in three patients with chronic airflow obstruction treated with "high doses" of beta adrenergic drugs in combination with high doses of oral corticosteroids and methylxanthines for long periods. ${ }^{8}$ Two of the patients died from left ventricular failure, the myocarditis being considered to be the main cause of death or a contributing factor.
Brittle asthma is difficult to treat effectively with conventional drugs and has a high mortality. 'We have obtained good control in these patients with continuous subcutaneous infusions of terbutaline via a portable infusion pump, or by subcutaneous injections of terbutaline every six hours. ${ }^{9}$ The effect is maintained with time. ${ }^{10}$

In view of the reports of myocarditis and the death of two of our patients while they were having continuous infusions of terbutaline, we set out to investigate whether long term subcutaneous terbutaline in these patients might have a deleterious effect on the myocardium.

\section{Methods}

SUBJECTS

We studied 12 patients (two male, 10 female), mean (SD) age 44 (14) years. All had brittle asthma (defined as a diurnal variation of greater than $40 \%$ on at least four days a week despite intensive medical treatment). Ten were receiving a continuous subcutaneous infusion of terbutaline and two subcutaneous terbutaline in divided doses. One further man, aged 65 , with non-brittle, steroid dependent asthma, who was also treated by a continuous infusion of terbutaline, was included. The mean (SD) daily dose of terbutaline was 9 (3) $\mathrm{mg}$, and the duration of treatment ranged from one week to four years. Seven of the patients with brittle asthma were also taking an oral methylxanthine and seven were taking oral corticosteroids; all were taking an inhaled beta $a_{2}$ agonist and an inhaled corticosteroid. The patient with non-brittle steroid dependent asthma was the only one with clinical evidence of ischaemic heart disease.

An age and sex matched group of patients with moderate but stable asthma (mean (SD) age 44 (15) years) acted as a control group. All the control patients were taking inhaled corticosteroids and an inhaled beta ${ }_{2}$ agonist, four an oral methylxanthine, and one an oral beta agonist.

Venous blood was taken for measurement of serum total creatine kinase and serum aspartate aminotransferase activity from the patients receiving subcutaneous terbutaline on at least two occasions and from the control group on one occasion. Blood was taken after the patient had rested for at least $\mathbf{3 0}$ minutes; 
no restriction had been placed on physical exertion in the previous 24 hours.

Three further patients were studied at the time they started treatment with a continuous subcutaneous infusion of terbutaline. The dose of subcutaneous terbutaline was increased every two days (saline first and then doses of 3,6 , and $12 \mathrm{mg} / 24$ hours). Blood was taken for estimation of serum total creatine kinase activity at admission and at the end of the second day of each treatment period.

\section{INVESTIGATIONS}

Creatine kinase and aspartate aminotransferase were determined as enzyme activities ${ }^{11}$ on a Hitatchi 705 analyser with reagents from BCL Ltd. The cardiac specific isoenzyme component of the total creatine kinase activity was measured by enzyme inhibition ${ }^{12}$ on serum stored at $-20^{\circ} \mathrm{C}$. The interassay coefficient of variation for total creatine kinase is $3 \%$.

A standard 12 lead electrocardiogram was recorded for each patient receiving subcutaneous terbutaline.

Electromyography of skeletal muscle using concentric needle electrodes was carried out on the five patients with brittle asthma receiving subcutaneous terbutaline with the highest total creatine kinase activity.

\section{ANALYSIS}

For the patients receiving subcutaneous terbutaline the mean of the two creatine kinase values was used in the analysis. Creatine kinase values in the two groups of patients were compared by paired rank sum test (Wilcoxon), $\mathrm{p}<0.05$ being taken as significant. Median values for each group were recorded as the data were not normally distributed.

\section{Results}

CREATINE KINASE ACTIVITY

The median total creatine kinase activity for the patients receiving subcutaneous terbutaline

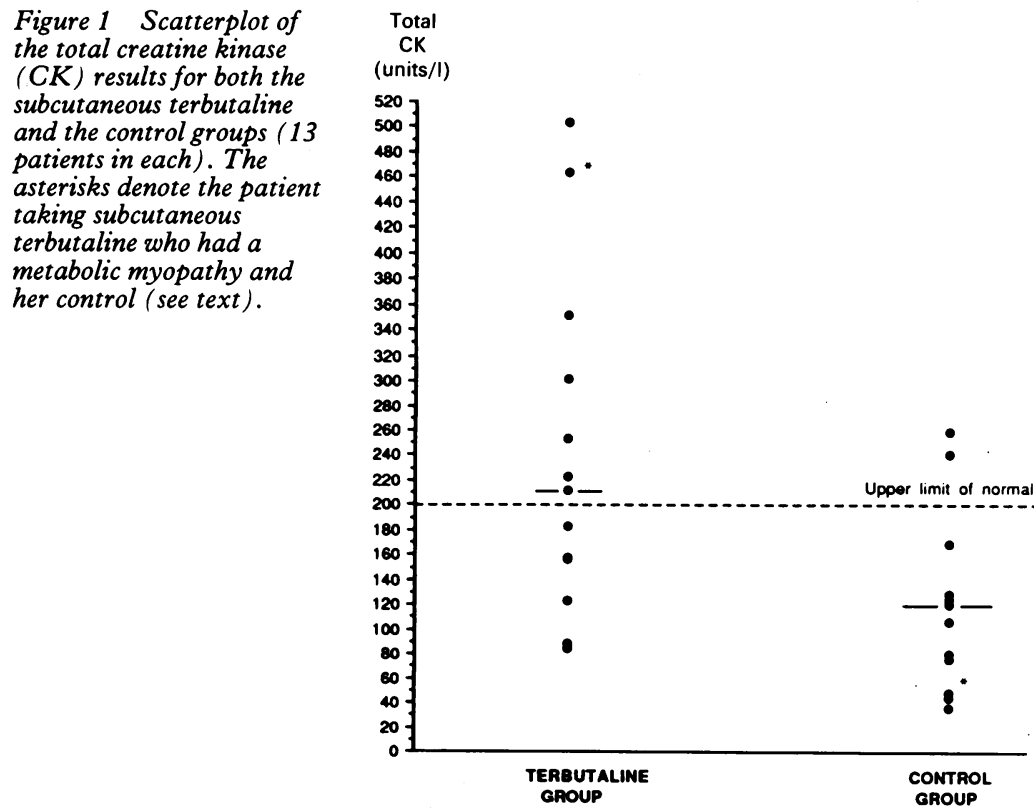

was 211 (range 84-503) units/1. Seven of these patients had values above the upper limit of normal (200 units/1), four of whom had values above 300 units/1 (fig 1). The control group had a median total creatine kinase activity of 120 units/1 (range 36-240 units/1); two patients had values slightly above the normal range (fig 1 ). The difference between the two groups was significant $(\mathrm{p}<0.005)$.

The cardiac specific isoenzyme of creatine kinase was determined in 11 of the patients with brittle asthma on subcutaneous terbutaline and in eight of the control group. The values were within the normal range ( 25 units/ 1) for all subjects in both groups, the median values being 15 for the subcutaneous terbutaline group and 11 for the control group $(\mathrm{p}=0.07)$. All aspartate aminotransferase values were within the normal range $(<35$ units/1).

A dose related increase in total creatine kinase was seen in the three patients with brittle asthma at the time that a continuous infusion of terbutaline was started (fig 2). Total creatine kinase did not increase after the infusion of saline (day 2).

\section{ELECTROCARDIOGRAMS}

Standard 12 lead electrocardiograms in the patients receiving subcutaneous terbutaline were normal. PR and QT intervals were in the normal range.

\section{ELECTROMYOGRAMS}

Of the seven patients receiving subcutaneous terbutaline who had a high creatine kinase value, five underwent electromyography, three of whom complained of non-specific myalgia.

Two of the five patients (both with myalgia) had changes compatible with diffuse myositis. One patient with a total creatine kinase activity of 463 units/1 had symptoms that predated the infusion of terbutaline. Subsequent investigations, including muscle biopsy and muscle biochemical analysis, showed a metabolic myopathy; the exact enzyme disorder was not defined. Her daughter has a similar problem and we believe that this may be a familial myopathy unrelated to asthma or its treatment. Reanalysis of the creatine kinase data excluding this patient and her control showed median values of 197 and 121 units/l for the subcutaneous terbutaline and control groups $(\mathrm{p}<0.02)$. The second patient with asthma was restudied after stopping his terbutaline infusion; total creatine kinase fell from 351 units/ 1 while he was taking terbutaline to 210 units/ 1 after he had stopped. The electromyogram abnormalities in this patient were unchanged. No further investigations have been made.

One patient receiving subcutaneous terbutaline in divided doses died from a severe acute attack of asthma. At necropsy the myocardium was normal with no histological evidence of focal myocardial necrosis. Skeletal muscle was not examined. 


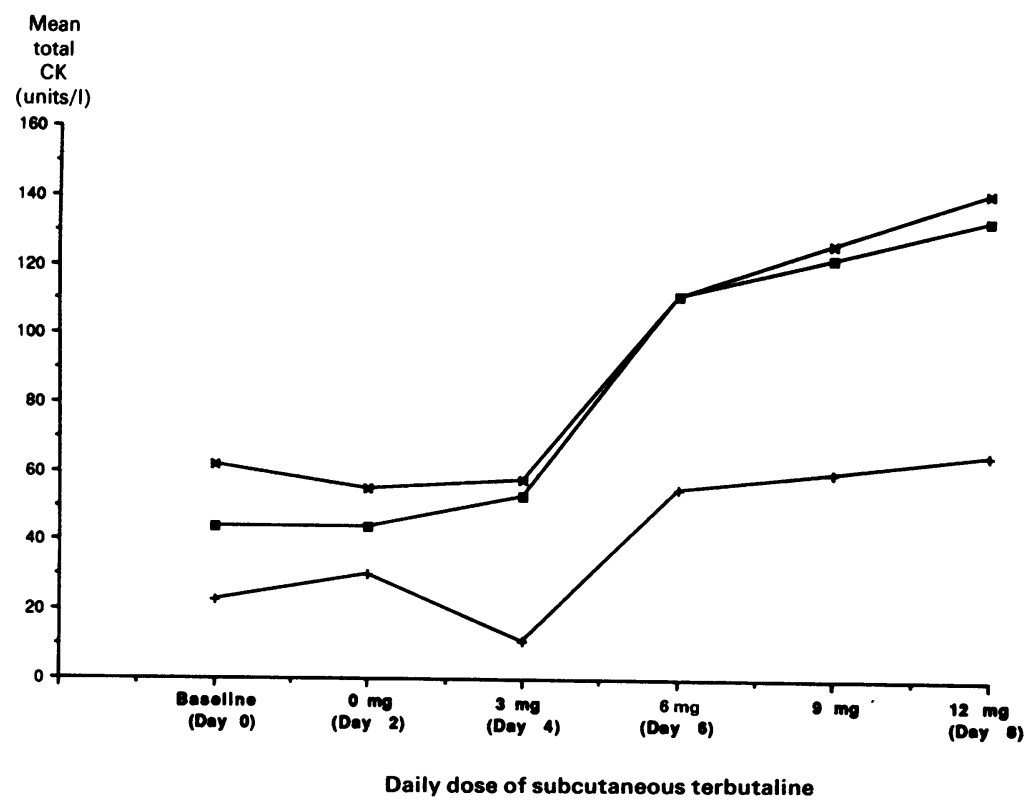

Figure 2 Total creatine kinase (CK) results measured at increasing terbutaline doses in 19 and 50 year old women and a 75 year old man being started on subcutaneous terbutaline treatment. sions, encephalitis, or meningitis, ${ }^{14}$ its level in our patients may be assumed to be negligible. The rise in total creatine kinase activity in our patients must therefore be due to the skeletal muscle specific isoenzyme.

Increased creatine kinase activity has been reported in patients with acute severe asthma, ${ }^{1516}$ possibly because of increased respiratory muscle activity. The report of raised creatine kinase activity in a patient performing yoga exercises during an asthma attack ${ }^{17}$ is of interest, but it is difficult to attribute the major cause of the rise. All our patients were studied in a stable condition and the dose-response effect seen in the three patients being started on a continuous infusion of terbutaline strongly suggests that the effect is related to terbutaline. The dose dependent rise in total creatine kinase could be a time dependent effect, but we think that this is unlikely as 48 hours was spent at each dose level, allowing time for a steady state to develop and, presumably, the effect on skeletal muscle. Although some of the patients were taking oral corticosteroids or oral methylxanthines or both, there was no relation between these preparations and total creatine kinase activity. The effect is therefore very unlikely to be due solely to either of these drugs.

The mechanism for the increase in the skeletal muscle specific isoenzyme with terbutaline is unknown, though tremor is a common side effect of terbutaline. Although large doses of terbutaline are used subcutaneously, the degree of tremor seen in these patients with asthma is very mild, and thus unlikely to account for the large differences in total creatine kinase between the two groups. Electromyogram recordings in two patients with brittle asthma were abnormal, confirming the presence of myositis, though in one this appears to be due to a longstanding metabolic myopathy. In the second patient the myositis appeared to be related to the start of subcutaneous terbutaline and it persisted after the treatment had been terminated, though to a lesser degree. The other three patients who had an electromyogram showed no evidence of myositis. These results suggest that terbutaline might cause myositis, but further work is required to determine the prevalence and clinical significance of this finding.

ferase activity were normal in the patients receiving subcutaneous terbutaline, which would support the suggestion that the treatment may not damage the myocardium. There was also no histological evidence of focal myocarditis in the patient who died while having subcutaneous terbutaline. Although myocarditis is usually associated with an increase in the cardiac specific isoenzyme component of creatine kinase or electrocardiogram changes, or both, these are not always seen and the degree of these changes will depend on the extent of tissue damage.

The rise in total creatine kinase activity must be due to the skeletal muscle specific isoenzyme or the brain specific isoenzyme. As the brain specific isoenzyme component of creatine kinase is normally very low, and increased only in cases of cerebral injury, brain tumour, convul-
APS is supplied by a National Asthma Campaign grant.

1 Szakacs JE, Cannon A. L-norepinephrine myocarditis. Am J Clin Pathol 1958;39:425-34.

2 Szakacs JE, Dimmette RM, Cowart EC. Pathological implication of the catecholamines, epinephrine and norepinephrine. US Armed Forces Med J1959;10:909-25.

3 Ferrans VJ, Hibbs RG, Walsh JJ, Burch GE. Histochemical and electron microscopic studies of the cardiac necrosis and electron microscopic studies of the cardiac necrosis 1969;156:309-32. catecholamine cardiomyopathy. Am Heart J 1985;109: 297-303.

5 Van Vliet PD, Burchell HB, Titus JL. Focal myocarditis associated with pheochromocytoma. N Engl J Med 1966;274:1102-8.

6 Kline IK. Myocardial alterations associated with pheochromocytomas. Am J Pathol 1961;38:539-57.

7 Kurland G, Williams J, Lewiston NJ. Fatal myocardial toxicity during continuous infusion intravenous
4 Simons $M$, Downing SE. Coronary vasoconstriction and 
isoproterenol therapy of asthma. J Allergy Clin Immunol 1979;63:407-11.

8 Nino AF, Berman MM, Gluck EH, Conway MM, Fisher JP Dougherty JE, et al. Drug-induced left ventricular failure in patients with pulmonary disease. Endomyocardial in patients with pulmonary disease. Endomyocardial
biopsy demonstration of catecholamine myocarditis. Chest biopsy demons

9 Ayres J, Fish DR, Wheeler DC, Wiggins J, Cochrane GM, Skinner C. Subcutaneous terbutaline and control of brittle asthma or appreciable morning dipping. $B M J 1984 ; 288$ : $1715-6$.

10 O'Driscoll BRC, Ruffles SP, Ayres Jon G, Cochrane GM Long term treatment of severe asthma with subcutaneous terbutaline. Br J Dis Chest 1988;82:360-7.

11 Szasz G, Gruber W, Bernt E. Creative kinase in serum: 1 Determination of optimum reaction conditions. Clin Chem 1976;22:650-6.
12 Wurzburg U, Hennrich $\mathrm{N}$, Lang $\mathrm{H}$, et al. Bestimmung der Aktivitat von Creatinkinase MB im Serum unter Verwendung inibierender Antikorper. Klin Wochenschr 1976;54:357-60

13 Sears MR, Taylor DR, Print CG, Lake DC, Li Q, Flannery $\mathrm{EM}$, et al. Regular inhaled beta-agonist treatment in bronchial asthma. Lancet 1990;336:1391-6.

14 Nevins MA, Saran M, Bright M, Lyon LJ. Pitfalls in interpreting serum creatine phosphokinase activity. JAMA 1973;224:1382-7.

15 Burki NK, Diamond L. Serum creatine phosphokinase activity in asthma. Am Rev Respir Dis 1977;116:327-31.

16 Karetzky MS. Blood studies in untreated patients with acute asthma. Am Rev Respir Dis 1975;112:607-13.

17 Tamarin FM, Conetta R, Brandstetter RD, Chadow H. Increased muscle enzyme activity after yoga breathing during an exacerbation of asthma. Thorax 1988;43:731-2. 